\title{
APPLICATION OF VARIATIONAL AND FEM METHODS TO THE MODELLING AND NUMERICAL ANALYSIS OF THE SLITTING PROCESS FOR GEOMETRICAL AND PHYSICAL NONLINEARITY
}

\author{
Łukasz Bohdal, Leon KukieŁka \\ Koszalin University of Technology, Koszalin, Poland \\ e-mail: bohdall@interia.pl; leon.kukielka@tu.koszalin.pl
}

\begin{abstract}
Finite element modelling provides a great deal of support in the understanding of technological processes. However, there are few studies of the slitting process, and those that exist are simplified for use only in the calculation of steady states of such processes. This paper proposes the application of variational and finite element methods for the analysis of slitting and the nonlinearities of this process. Physical and mathematical models of the process and a new thermo-elastic/thermo-visco-plastic material model are elaborated. The procedure is implemented in the finite element code ANSYS/LS-DYNA and the model is validated comparing the numerical and experimental results. The influence of various process conditions on the strain and stress states and the quality of the final product are analysed. The results lay the groundwork for further study regarding the numerical analysis of spring-back behaviour and the effect of tool elasticity on the quality of the final workpiece.
\end{abstract}

Keywords: slitting, constitutive laws, modelling, numerical simulation, variational formulation, FEM

\section{Introduction}

Slitting is a sheet metal-, metal alloy- or magnetic tapes-cutting process that uses circular knives. Industries use slitting to split wide coiled sheets into narrower widths or for the edge trimming of rolled sheets (Lu et al., 2001; Liu et al., 2005). This process is schematically drawn in Fig. 1a. The sheet is first plastically deformed (A-B). Continued deformation leads to ductile fracture (B-C) and complete separation of the parts (D) (Wisselink, 2000; Gałęzia et al., 2012). The quality of the part after slitting depends on many process parameters such as clearance between the knives in the horizontal and vertical direction, lubrication method, shearing velocity, knife geometry and the method of clamping the sheet plates (Meehan and Burns, 1998; Ma et al., 2006). These parameters are key factors affecting energy consumption and physical phenomena occurring in the cutting process such as the state of material displacement, visco-plastic strain and stress. In turn, certain physical phenomena affect the mechanisms of separation of the material in the cutting zone, such as visco-plastic shear or rupture, because the mechanism of separation depends on the quality of both the sheared edge and the product. The amount of adjustable process parameters and the fact that the influence of these parameters on the process are not fully understood, makes it difficult to control the slitting process. In practice, the right setup for the slitters is mostly found by a trial and error method combined with the experience. Therefore, the final product frequently has serious defects and drawbacks such as large deformation and defects on the sheared edge, such as burrs and edge wave (Fig. 1b).

Computational models, such as the finite element method (FEM), are valuable in reducing the number of trial-and-error experiments required to predict the state of material displacement, residual stresses, strains, material fracture, sheet deformations and quality of the sheared edge. 

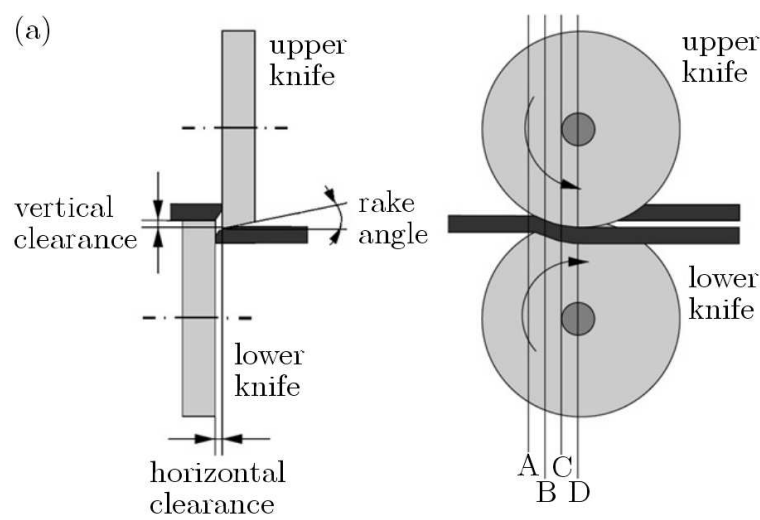

Fig. 1. (a) Scheme of the slitting process; (b) typical defects in the sheet sheared edge (b)
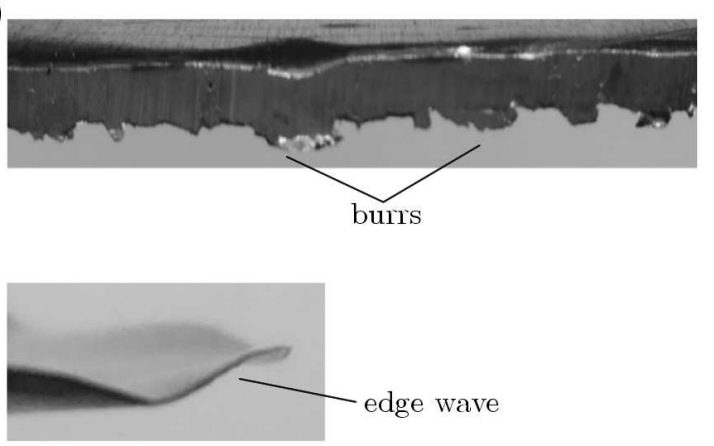

Some of the researches have studied the process using FEM. Wisselink and Huetink (2004) developed a finite element model of slitting to calculate the steady state of such a process using the Arbitrary Lagrangian Eulerian formulation. An isotropic plastic constitutive equation combined with a simple uncoupled damage model was used to describe the material behaviour. This model was based on stress triaxiality, which was defined as the ratio of the hydrostatic stress to the equivalent stress. The simulations performed as a part of the study completely neglected the effect of damage on the material elastic and plastic behaviour and the effects of strain rate and temperature-dependent material properties. The developed FE model was used to simulate residual sheet stresses and deformation, though. Meehan and Burns (1998) studied the slitting using photoelastic micrographs and made observations of two-dimensional stress distributions. Aggarwal et al. (2005) conducted a finite element analysis of the magnetic tape slitting process. The polymeric tape substrate was modeled using a pressure dependent yield function and the material separation was incorporated using the shear failure criterion. Saanouni (2006) coupled Continuum Damage Mechanics with an FE model describing elastoplastic behaviour to numerically simulate the slitting of a metallic sheet. An implicit NewtonRaphson scheme, together with a dynamic explicit resolution strategy, was used to analyse the effects of process configurations on the state of stress in the sheet.

Analysis of the current literature suggests that the main difficulty in the modelling of the slitting is that only a limited number of FEM models are currently capable of describing the complete shearing process, including the complete separation of the material parts through ductile fracturing. A model describing the primary physical phenomena and characterising the mechanical behaviour of the metal sheet is required to numerically simulate this process using finite element analysis. If the modelling used the updated Lagrangian formulation, FEM considering process nonlinearity (geometrical, physical, and thermal), large deformations, strain rate, friction and nonlinear material characteristics could be used to analyse the thermo-visco-plastic flow of the material and its stress and strain fields at any time during the slitting process, successfully numerically reproducing the operation. The slitting differs from the other shearing operations such as blanking and punching as it involves the rotation of blades and, hence, the material is cut in two directions simultaneously instead of one. A thorough understanding of the process of slitting would thus require a three-dimensional analysis of the process to be conducted. The three-dimensional analysis would allow a complete study of the process enabling the consideration of parameters such as blade geometry, knife radius, shearing velocity, etc., which cannot be studied using a simplified two-dimensional model.

In this paper, the slitting process is considered as an initial and boundary value problem with geometrical, physical and thermal nonlinearity and with only partial knowledge of the boundary conditions. The boundary conditions in the tools-sheet contact zones are not known. The paper 
focuses on the development of a methodology to study the phenomena that occur during and after the cutting process (e.g., the change from the steady state to the unsteady state, sheet deformation, stress and strain states and the sheared edge characteristic features). Physical and mathematical models of the slitting process and a new thermo-elastic/thermo-visco-plastic material model are elaborated. The updated Lagrange description is used to describe nonlinear phenomena on a typical incremental step using a stepwise and co-rotational coordinate system. The strain and strain rate states are described using Green-Lagrange strain and strain rate tensors, respectively, and are nonlinear dependences without any linearisation. The procedure is employed in an application created by the authors using ANSYS/LS-DYNA program and the finite element method. The study presents sample 3D simulations and experimentally verifies and analyses the impact of various process conditions on the material displacements, stresses, strains and quality of the final product.

\section{Thermo-elastic and thermo-visco-plastic material model}

The goal of this Section is to present a material model that considers the combined effects of thermo-elasticity (in the reversible zone) and thermo-visco-plasticity (in the non-reversible zone). The model accounts for the strain, strain rate and material temperature histories. The formulation of the model assumes that the typical small incremental components $\Delta \varepsilon_{i j}$ of the strain tensor $\mathbf{T}_{\Delta \varepsilon}$ can be expressed as the sum of thermo-elastic $\Delta \varepsilon_{i j}^{(T E)}$, visco-plastic $\Delta \varepsilon_{i j}^{(V P)}$ and thermal $\Delta \varepsilon_{i j}^{(T H)}$ incremental strains

$$
\Delta \varepsilon_{i j}=\Delta \varepsilon_{i j}^{(T E)}+\Delta \varepsilon_{i j}^{(V P)}+\Delta \varepsilon_{i j}^{(T H)}
$$

where: $\Delta \varepsilon_{i j}, \Delta \varepsilon_{i j}^{(T E)}, \Delta \varepsilon_{i j}^{(V P)}$ and $\Delta \varepsilon_{i j}^{(T H)}$ are the incremental components of the total, thermoelastic, visco-plastic and thermal strain tensors, respectively.

The constitutive law for an isotropic, thermo-elastic material with a temperature-dependent modulus is

$$
\Delta \sigma_{i j}=C_{i j k l}^{(T E)}\left(\Delta \varepsilon_{k l}-\Delta \varepsilon_{k l}^{(V P)}-\Delta \varepsilon_{k l}^{(T H)}\right)+\Delta C_{i j k l}^{(T E)} \varepsilon_{k l}^{(E)}
$$

where: $\Delta \sigma_{i j}$ are the incremental components of the second Piola-Kirchhoff stress, $\Delta \varepsilon_{i j}$ are the incremental components of the Green-Lagrange strain tensor, $C_{i j k l}^{(T E)}(T)=\lambda(T) \delta_{i j} \delta_{k l}+$ $\mu(T)\left(\delta_{i k} \delta_{j l}+\delta_{i l} \delta_{j k}\right)$ is the component of the temperature-dependent elastic constitutive tensor, $\Delta C_{i j k l}^{(T E)}(\Delta T)=\left[\partial C_{i j k l}^{(T E)}(T) / \partial T\right] \Delta T$ are its increments

$$
\lambda(T)=\frac{E(T) \nu(T)}{[1+\nu(T)][1-2 \nu(T)]} \quad \mu(T)=\frac{E(T)}{2[1+\nu(T)]}
$$

$E(T)$ and $\nu(T)$ are temperature-dependent Young's modulus and Poisson's ratio, respectively, $\delta_{i j}$ is the Kronecker delta and $\varepsilon_{k l}^{(E)}$ are the accumulated components of the elastic strain tensor at the time $t$. The components of the thermal increment strain tensor are calculated from the equation

$$
\Delta \varepsilon_{i j}^{(T H)} \cong \alpha_{m}(T) \Delta T \delta_{i j}
$$

where $\alpha_{m}$ is the mean coefficient of thermal expansion. The visco-plastic incremental strain is defined as (Kleiber, 1985)

$$
\Delta \varepsilon_{i j}^{(V P)}=\Delta \lambda \frac{\partial F}{\partial \widetilde{S}_{i j}}
$$


where $\Delta \lambda$ is the positive scalar variable or Lagrange's coefficient and $\widetilde{S}_{i j}=S_{i j}-\alpha_{i j}$ is the component of the reduced stress deviator $\widetilde{\mathbf{D}}_{\sigma}$.

The calculation of $\Delta \lambda$ requires a hardening rule to be selected. The hardening rule describes the change in the yield surface with continuing plastic deformation.

For mixed hardening and an isotropic body, the rule is defined by

$$
\Delta \lambda=\frac{\widetilde{S}_{i j} C_{i j k l}^{(T E)}\left(\Delta \varepsilon_{k l}^{(T E)}+\Delta \varepsilon_{k l}^{(V P)}\right)+\widetilde{S}_{i j} \Delta C_{i j k l}^{(T E)} \varepsilon_{k l}^{(E)}-A}{\widetilde{S}_{i j} C_{i j k l}^{(T E)} \widetilde{S}_{k l}+\frac{2}{3} \sigma_{Y}^{2}\left(\widetilde{C}+\frac{2}{3} E_{T}\right)}
$$

where

$$
A=\frac{2}{3} \sigma_{Y}\left(\varepsilon_{i}^{(V P)}, \dot{\varepsilon}_{i}^{(V P)}, T\right)\left(\dot{E}_{T} \Delta \dot{\varepsilon}_{i}^{(V P)}+T_{T} \Delta T\right)
$$

is the positive scalar variable, $\tan \alpha=E_{T}(T)=\partial \sigma_{Y} / \partial \varepsilon_{i}^{(V P)}$ is the hardening modulus at $T$, $\tan \beta=\dot{E}_{T}=\partial \sigma_{Y} / \partial \dot{\varepsilon}_{i}^{(V P)}$ is the change in temporary yield stress with a change in the intensity strain rate, $\tan \gamma=T_{T}=\partial \sigma_{Y} / \partial T$ is the change of temporary yield stress with a change in temperature, $\widetilde{C}(T)=\left[2 E(T) E_{T}(T)\right] /\left[3 E(T)-E_{T}(T)\right]$ is the kinematic hardening parameter and $E$ is Young's modulus at the temperature $T$. To further evaluate the above expression for $\Delta \lambda$, it is necessary to obtain the partial derivatives $\partial \sigma_{Y} / \partial \varepsilon_{i}^{(V P)}, \partial \sigma_{Y} / \partial \dot{\varepsilon}_{i}^{(V P)}$ and $\partial \sigma_{Y} / \partial T$. It is assumed that the relationship between $\sigma_{Y}, \varepsilon_{i}^{(V P)}, \dot{\varepsilon}_{i}^{(V P)}$ and $T$ can be derived from the data obtained in a series of tensile tests at different temperatures and strain rates using virgin material specimens.

Substituting equation (2.5) into equation (2.4) and using $\partial F / \partial \widetilde{S}_{i j}=\widetilde{S}_{i j}$, we obtain

$$
\Delta \varepsilon_{i j}^{(V P)}=\widetilde{S}^{* *} \Delta \varepsilon_{i j}+\Delta \varepsilon_{i j}^{* *}
$$

where

$$
\widetilde{S}^{* *}=\widetilde{S}_{i j}^{*} C_{i j m n}^{(T E)} \widetilde{S}_{m n}
$$

is a positive scalar variable

$$
\Delta \varepsilon_{i j}^{* *}=\widetilde{S}_{i j}^{*}\left(-C_{i j m n}^{(T E)} \widetilde{S}_{m n} \alpha_{m} \Delta T \delta_{i j}+\widetilde{S}_{m n} \Delta C_{m n k l}^{(T E)} \varepsilon_{k l}^{(E)}-A\right)
$$

is the component of the incremental strain tensor, and

$$
\widetilde{S}_{i j}^{*}=\frac{\widetilde{S}_{i j}}{\widetilde{S}_{i j} C_{i j k l}^{(T E)} \widetilde{S}_{k l}+\frac{2}{3} \sigma_{Y}^{2}\left(\widetilde{C}+\frac{2}{3} E_{T}\right)}
$$

is the component of the stress tensor.

Substituting equations (2.3) and (2.7) into equation (2.2), we obtain the constitutive equation of mixed hardening for an isotropic material that includes the combined effects of thermoelasticity and thermo-visco-plasticity

$$
\Delta \sigma_{i j}=C_{i j k l}^{(T E)}\left(1-\widetilde{S}^{* *}\right) \Delta \varepsilon_{k l}-C_{i j k l}^{(T E)}\left(\Delta \varepsilon_{k l}^{* *}+\alpha_{m} \Delta T \delta_{k l}\right)+\Delta C_{i j k l}^{(T E)} \varepsilon_{k l}^{(E)}=C_{i j k l}^{(T E) *} \Delta \varepsilon_{k l}+\Delta \sigma_{i j}^{* *}
$$

where

$$
\begin{aligned}
& C_{i j k l}^{(T E) *}=C_{i j k l}^{(T E)}\left(1-\widetilde{S}^{* *}\right) \\
& \Delta \sigma_{i j}^{* *}=-C_{i j k l}^{(T E)}\left(\Delta \varepsilon_{k l}^{* *}+\alpha_{m} \Delta T \delta_{k l}\right)+\Delta C_{i j k l}^{(T E)} \varepsilon_{k l}^{(E)}
\end{aligned}
$$




\section{Damping models}

A very essential and difficult problem during the modelling of technological processes is consideration of the phenomenon of energy dissipation (Gontarz and Radkowski, 2012). The dissipation of energy influences the decrease of displacement and dynamic stress in the object. The difficulty depends on selection of the proper physical model mainly as well as qualification of suitable values of its parameters. At present (Bathe, 1982), usually the Rayleigh damping is used, in which the damping matrix $\mathbf{C}$ is calculated from the equation: $\mathbf{C}=\alpha_{1} \mathbf{M}+\alpha_{2} \mathbf{K}$, where $\mathbf{M}$ and $\mathbf{K}$ are mass and stiffness matrices, $\alpha_{1}$ and $\alpha_{2}$ are constants to be determined from two given damping ratios that correspond to two unequal frequencies of vibration. In the case of the modelling of dynamical processes, one should apply damping models dependent on velocity, strain and strain rate. For this reason, we propose to calculate the dissipation of energy, for a typical step time, from the following expression

$$
\begin{aligned}
\Delta E_{d} & \cong \alpha_{1} \int_{V} \rho \Delta \dot{u}_{i} \Delta u_{i} d V+\alpha_{1} \int_{V} \rho \Delta \dot{u}_{i} u_{i} d V+\alpha_{1} \int_{V} \rho \dot{u}_{i} \Delta u_{i} d V+\frac{1}{2} \alpha_{2} \int_{V} C_{i j k l}^{(T E)} \Delta \dot{\varepsilon}_{i j} \Delta \varepsilon_{k l} d V \\
& +\frac{1}{2} \alpha_{2} \int_{V} C_{i j k l}^{(T E)} \Delta \dot{\varepsilon}_{i j} \varepsilon_{k l} d V+\frac{1}{2} \alpha_{2} \int_{V} C_{i j k l}^{(T E)} \dot{\varepsilon}_{i j} \Delta \varepsilon_{k l} d V
\end{aligned}
$$

where: $\Delta u_{i}, \Delta \dot{u}_{i}$ are the $i$-th increment components of displacement and velocity vectors at the typical step time $\Delta t$, respectively, $u_{i}$ is the accumulated $i$-th component of the displacement vector at the time $t$.

Constitutive models (2.11) and (3.1) can be used for practical engineering analysis. In the next Section, these models are used for variational formulation of a nonlinear equation of motion for the slitting operation.

\section{Variational formulation of the equation of motion}

In this Section, we develop the equation of motion for a three-dimensional body in the global Cartesian coordinates $\{z\}$ and the updated Lagrange incremental formulation. Variational methods are used in continuum mechanics to formulate the equations of motion (Kleiber, 1985; Orłowska and Czapp, 2012). However, there is no method of accounting for the incremental formulation. The use of the variational method to formulate the equation of motion for a typical incremental step in nonlinear problems in technological processes was proposed by Kulakowska and Kukielka (2008), Kukielka et al. (2012). In this paper, this method is used to model the slitting process. Assuming that a numerical solution has been obtained at discrete time points $\Delta t, 2 \Delta t, \ldots$, the solution for $t+\Delta t$ is desired. In this case, the functional increment is formulated for the incremental displacement $\Delta \mathbf{F}\left(\Delta \ddot{u}_{i}, \Delta \dot{u}_{i}, \Delta u_{i}\right)=\Delta \mathbf{F}(\cdot)$, where $\Delta u_{i}, \Delta \dot{u}_{i}, \Delta \ddot{u}_{i}$ are the incremental components of the displacement, velocity and acceleration vectors, respectively. Using the conditions for the functional $\Delta \mathbf{F}(\cdot)$ being stationary (Kukielka et al., 2012)

$$
\delta[\Delta \mathbf{F}(\cdot)]=\frac{\partial[\Delta \mathbf{F}(\cdot)]}{\partial\left(\Delta u_{i}\right)} \delta\left(\Delta u_{i}\right)=\frac{\partial[\Delta \mathbf{F}(\cdot)]}{\partial\left(\Delta u_{i}\right)} \Delta_{\underline{u}_{i}}
$$

where the underline $(\cdot)$ denotes "variation in", and because $\Delta u_{i}$ is the only variable, we obtain

$$
\begin{aligned}
& \delta[\Delta \mathbf{F}(\cdot)]=\int_{V} \rho\left(\ddot{u}_{i}+\Delta \ddot{u}_{i}\right) \Delta \underline{u}_{i} d V+\alpha_{1} \int_{V} \rho \Delta \dot{u}_{i} \Delta \underline{u}_{i} d V-2 \omega \int_{V} \rho \Delta \dot{u}_{i} \Omega_{i j} \Delta \underline{u}_{j} d V \\
& +\frac{1}{2} \alpha_{2} \int_{V} \Delta \dot{\widetilde{\varepsilon}}_{i j} C_{i j k l}^{(T E)} \Delta \dot{\varepsilon}_{k l} d V+\frac{1}{2} \alpha_{2} \int_{V} \Delta \dot{\widetilde{\varepsilon}}_{i j} C_{i j k l}^{(T E)} \Delta \underline{\bar{\varepsilon}}_{k l} d V+\frac{1}{2} \alpha_{2} \int_{V} \Delta \dot{\bar{\varepsilon}}_{i j} C_{i j k l}^{(T E)} \Delta \underline{\widetilde{\varepsilon}}_{k l} d V
\end{aligned}
$$




$$
\begin{aligned}
& +\frac{1}{2} \alpha_{2} \int_{V} \Delta \dot{\bar{\varepsilon}}_{i j} C_{i j k l}^{(T E)} \Delta \underline{\varepsilon}_{k l} d V+\frac{1}{2} \alpha_{2} \int_{V} \Delta \dot{\widetilde{\varepsilon}}_{i j} C_{i j k l}^{(T E)} \Delta \widetilde{\varepsilon}_{k l} d V+\frac{1}{2} \alpha_{2} \int_{V} \Delta \underline{\widetilde{\varepsilon}}_{i j} C_{i j k l}^{(T E)} \Delta \bar{\varepsilon}_{k l} d V \\
& +\frac{1}{2} \alpha_{2} \int_{V} \Delta \dot{\bar{\varepsilon}}_{i j} C_{i j k l}^{(T E)} \Delta \widetilde{\varepsilon}_{k l} d V+\frac{1}{2} \alpha_{2} \int_{V} \Delta \underline{\dot{\varepsilon}}_{i j} C_{i j k l}^{(T E)} \Delta \bar{\varepsilon}_{k l} d V+\int_{V} \Delta \widetilde{\varepsilon}_{i j} C_{i j k l}^{(T E) *} \Delta \underline{\widetilde{\varepsilon}}_{k l} d V \\
& +\int_{V} \Delta \widetilde{\varepsilon}_{i j} C_{i j k l}^{(T E) *} \Delta \underline{\varepsilon}_{k l} d V+\int_{V} \Delta \bar{\varepsilon}_{i j} C_{i j k l}^{(T E) *} \Delta \underline{\widetilde{\varepsilon}}_{k l} d V+\int_{V} \Delta \bar{\varepsilon}_{i j} C_{i j k l}^{(T E) *} \Delta \bar{\varepsilon}_{k l} d V \\
& +\frac{1}{2} \int_{V}\left(T_{i j}+\Delta \sigma_{i j}^{* *}\right) \Delta \underline{\widetilde{\varepsilon}}_{i j} d V+\frac{1}{2} \int_{V}\left(T_{i j}+\Delta \sigma_{i j}^{* *}\right) \Delta \underline{\varepsilon}_{i j} d V-\omega^{2} \int_{V} \rho \Delta u_{i} \Omega_{i j} \Omega_{i j} \Delta \underline{u}_{i} d V \\
& -\omega^{2} \int_{V} \rho r_{i} \Omega_{i j} \Omega_{i j} \Delta \underline{u}_{i} d V-\int_{V} \rho\left(f_{i}+\Delta f_{i}\right) \Delta \underline{u}_{i} d V-\int_{\Sigma_{k}}\left(\widehat{p}_{i}+\Delta \widehat{p}_{i}\right) \Delta \underline{u}_{i} d \Sigma_{k}=0
\end{aligned}
$$

where: $T_{i j}$ is the component of Cauchy's stress tensor, $\Delta \dot{\bar{\varepsilon}}_{i j}$ and $\Delta \dot{\widetilde{\varepsilon}}_{i j}$ are the linear and nonlinear incremental components of the Green-Lagrange strain rate tensor, respectively $\left(\Delta \dot{\bar{\varepsilon}}_{i j}=\partial \Delta \bar{\varepsilon}_{i j} / \partial t ; \Delta \dot{\widetilde{\varepsilon}}_{i j}=\partial \Delta \widetilde{\varepsilon}_{i j} / \partial t, \Delta \bar{\varepsilon}_{i j}=\left(\Delta u_{i, j}+\Delta u_{j, i}+u_{l, i} \Delta u_{l, j}+\Delta u_{l, i} u_{l, j}\right) / 2\right.$ and $\left.\Delta \widetilde{\varepsilon}_{i j}=\left(\Delta u_{i, k} \Delta u_{j, k}\right) / 2\right), \rho(T)$ is the temperature-dependent mass density at the time $t$ and $\varepsilon_{i j}$ are the accumulated components of the total strain tensors at the time $t$ (depending on the history of deformation and temperature). $f_{i}, \Delta f_{i}$ are the components of the internal force and incremental force vectors, respectively, $\widehat{p}_{i}, \Delta \widehat{p}_{i}$ are the components of the externally applied surface force and surface incremental force vectors in the contact body zones, respectively, and $\Omega_{i j}$ are the components of the gyro tensor. However, in the typical updated Lagrange formulation $\Delta \bar{\varepsilon}_{i j}=\left(\Delta u_{i, j}+\Delta u_{j, i}\right) / 2$. The integrations are performed over the volume $V$ and surface $\Sigma$ of the body, and equation (4.2) provides the basis for the finite element discretisation required to obtain the solution.

\section{Numerical analysis algorithm}

Assuming that the temporary step time $\Delta t$ is very small, it is possible to remove the incremental stiffness matrix $(\Delta \mathbf{K} \approx \mathbf{0})$ and the internal incremental force vector $(\Delta \mathbf{F} \approx \mathbf{0})$. Then, using the principle of incremental decomposition, approximating $\dot{\mathbf{r}}$ and $\ddot{\mathbf{r}}$ in terms of $\mathbf{r}$ in equation (4.2) using the central difference method (DEM), and using the following approximate (Bathe, 1982)

$$
{ }^{t} \dot{\mathbf{r}}=\frac{1}{2 \Delta t}\left({ }^{\tau} \mathbf{r}-{ }^{t-\Delta t} \mathbf{r}\right) \quad{ }^{t} \ddot{\mathbf{r}}=\frac{1}{\Delta t^{2}}\left({ }^{\tau} \mathbf{r}-2{ }^{t} \mathbf{r}+{ }^{t-\Delta t} \mathbf{r}\right)
$$

we obtain the solution of ${ }_{t}^{\tau} \mathbf{r}$

$$
\widetilde{\mathbf{M}}{ }_{t}^{\tau} \mathbf{r}=\mathbf{Q}
$$

where $\widetilde{\mathbf{M}}$ is the effective mass matrix and $\widetilde{\mathbf{Q}}$ the effective load vector

$$
\begin{aligned}
& \widetilde{\mathbf{M}}=a_{0} \mathbf{M}+a_{1} \mathbf{C} \\
& \widetilde{\mathbf{Q}}=\mathbf{F}+\mathbf{R}+a_{0} \mathbf{M}\left(2^{t} \mathbf{r}-{ }^{t-\Delta t} \mathbf{r}\right)+a_{1} \mathbf{C}^{t-\Delta t} \mathbf{r}
\end{aligned}
$$

with the integration constants $a_{0}=1 /\left(\Delta t^{2}\right), a_{1}=1 /(2 \Delta t)$.

\subsection{Step-by-step solution using the Central Difference Method}

In this Section, the Central Difference Method (Bathe, 1982; Patyk and Kukielka, 2008) is adopted to solve Eq. (5.2) with initial and boundary conditions. 
- Initial calculations:

1. Form the mass $\mathbf{M}$ and damping $\mathbf{C}$ matrices.

2. Initialise ${ }^{0} \mathbf{r},{ }^{0} \dot{\mathbf{r}}$ and ${ }^{0} \ddot{\mathbf{r}}$.

3. Select a time step $\Delta t<\Delta t_{c r}=T_{N} / \pi$ and calculate the integration constants $a_{0}$ and $a_{1}$.

4. Calculate ${ }^{-{ }^{-}} \mathbf{r}$.

5. Form the effective stiffness matrix $\widetilde{\mathbf{M}}$ from equation (5.3).

- For each time step:

1. Calculate the effective load vector $\widetilde{\mathbf{Q}}$ from equation (5.4).

2. Partitioning the equilibrium equation for two blocks, write the problem in form

$$
\left[\begin{array}{ll}
\widetilde{\mathbf{M}}_{11}^{n \times n} & \widetilde{\mathbf{M}}_{12}^{n \times w} \\
\widetilde{\mathbf{M}}_{21}^{w \times n} & \widetilde{\mathbf{M}}_{22}^{w \times w}
\end{array}\right]\left\{\begin{array}{c}
{ }_{t}^{\tau} \mathbf{r}_{1}^{n \times 1} \\
{ }_{t} \mathbf{r}_{2}^{w \times 1}
\end{array}\right\}=\left\{\begin{array}{c}
\widetilde{\mathbf{Q}}_{1}^{n \times 1} \\
\widetilde{\mathbf{Q}}_{2}^{w \times 1}
\end{array}\right\}
$$

where the vectors $\mathbf{r}_{2}^{w \times 1}, \widetilde{\mathbf{Q}}_{1}^{n \times 1}$ are known, and the vectors $\mathbf{r}_{1}^{n \times 1}, \widetilde{\mathbf{Q}}_{2}^{w \times 1}$ are unknown.

3. Solve for the displacement vector ${ }_{t}^{\tau} \mathbf{r}_{1}^{n \times 1}$

$$
{ }_{t}^{\tau} \mathbf{r}_{1}^{n \times 1}=\left(\widetilde{\mathbf{M}}_{11}^{n \times n}\right)^{-1}\left[\widetilde{\mathbf{Q}}_{1}-\widetilde{\mathbf{M}}_{12}^{n \times w}{ }_{t}^{\tau} \mathbf{r}_{2}^{w \times 1}\right]
$$

4. Substitute the vector ${ }_{t}^{\tau} \mathbf{r}_{1}^{n \times 1}$ into the equation

$$
\widetilde{\mathbf{Q}}_{2}^{w \times 1}=\widetilde{\mathbf{M}}_{21}^{w \times n}{ }_{t}^{\tau} \mathbf{r}_{1}^{n \times 1}+\widetilde{\mathbf{M}}_{22}^{w \times w}{ }_{t}^{\tau} \mathbf{r}_{2}^{w \times 1}
$$

and solve it to obtain the load vector $\widetilde{\mathbf{Q}}_{2}^{w \times 1}$ in the tool-object contact zone.

5. Calculate the vectors ${ }^{t} \dot{\mathbf{r}}$ and ${ }^{t} \ddot{\mathbf{r}}$ from equation (5.1).

\section{Sample solution}

A three-dimensional finite element model of the slitting is presented in Fig. 2a. The model is created based on the experimental configuration and the geometrical parameters of our test stand shown in Fig. 2b. The slitting machine (KSE 10/10) consists of two rotary knives driven by the engine.

The contact between the knives and sheet is considered a non-sliding contact that uses a polyurethane roll, which moves the sheet in the horizontal direction. The sheet is clamped by a sheet holder, and a rake angle $\left(\alpha=7^{\circ}\right)$ is applied to the upper knife. The radius of the upper knife carry out $14 \mathrm{~mm}$, the radius of the lower knife is $12 \mathrm{~mm}$. The width of the knives carry out $15 \mathrm{~mm}$. Numerical calculations are performed for the 3D state of strain and 3D state of stress in the FE model. It is assumed that the cutting process takes place under a constant temperature $(\Delta T=0)$. The models in $(2.11)$ and $(4.2)$ reduce then to the elastic (in the reversible zone) and visco-plastic (in the non-reversible zone) strains. Moreover: $\lambda(T)=\lambda, \mu(T)=\mu, \rho(T)=\rho$, $\Delta \varepsilon_{i j}^{(T H)}=0, \Delta C_{i j k l}^{(T E)}(\Delta T)=0, C_{i j k l}^{(T E)}=C_{i j k l}^{(E)}$ and $\Delta \sigma_{i j}^{* *}=-C_{i j k l}^{(T E)} \Delta \varepsilon_{k l}^{* *}$. Aluminum alloy AA6111-T4, which is often employed for exterior panels in the automotive industry, is used to simulate typical production conditions. An element with length of $l=50 \mathrm{~mm}$, width of $w_{i}=30 \mathrm{~mm}$ and thickness of $t=1.5 \mathrm{~mm}$ is analysed. The kinematics of different components is as follows: first the upper knife and the lower one moves vertically in opposite directions with the same constant velocity $v_{1}=200 \mathrm{~mm} / \mathrm{s}$ in order to cut the sheet. Then, the sheet moves along the 

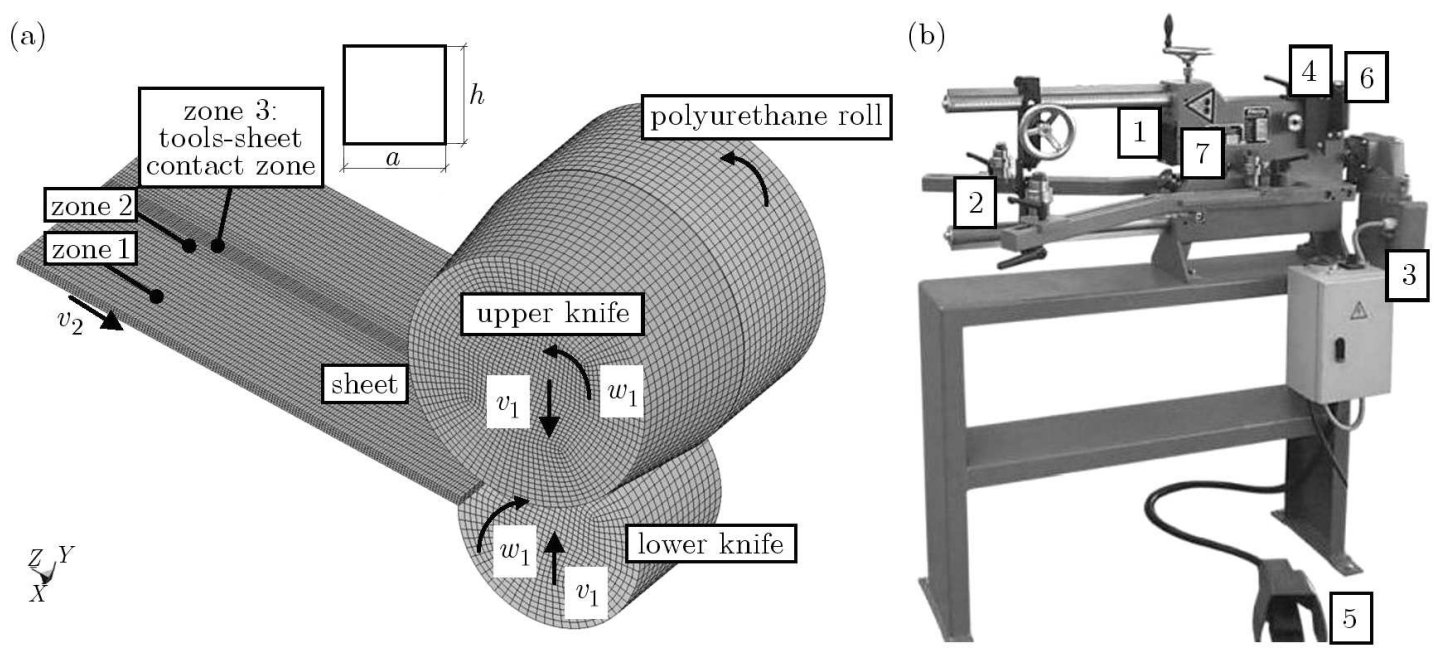

Fig. 2. (a) Scheme of the 3D FE model of the slitting process; (b) schematic view of the experimental slitting equipment: 1 - knives, 2 - sheet holder, 3 - engine, 4 - clearance regulator, 5 - drive pedal, 6 - slitting velocity regulator, 7 - force sensor

$Z$ axis with a constant velocity $v_{2}=500 \mathrm{~mm} / \mathrm{s}$, and at the same time the upper knife and roll rotates with a angular velocity $w_{1}=36 \mathrm{rad} / \mathrm{s}$ and the lower knife rotate in the opposite direction with the same angular velocity. The value of horizontal clearance carry out $c=0.1 \mathrm{~mm}$. The objects are meshed with an 8-node Solid164 element type with reduced integration and hourglass control, and the mapped mesh with various densities is generated in three areas on the sheet (Fig. 2a). The size of elements (where a is defined as element length, and $\mathrm{h}$ is defined as element height) in each zone is as follows: in zone 1: $a=0.8 \mathrm{~mm}, h=0.37 \mathrm{~mm}$, in zone $2: a=0.5 \mathrm{~mm}$, $h=0.37 \mathrm{~mm}$, in zone 3: $a=h=0.1 \mathrm{~mm}$. The finite element model of the sheet uses 320000 elements, that of the upper knife uses 27648, that of the lower knife uses 11520 and that of the roll uses 25920. The contact between the tools and the deformable sheet metal is described using Coulomb's friction model, and constant coefficients of static friction $\mu_{s}=0.08$ and kinetic friction $\mu_{d}=0.009$ are accepted.

The tools (knives) are considered as rigid bodies, but the sheet is modelled as an isotropic, elastic/visco-plastic material with nonlinear hardening the temporary yield stress of which is described with the aid of the Cowper-Symonds model (Bohdal and Kukielka, 2006)

$$
\sigma_{Y}=\left(1+\frac{\dot{\varepsilon}_{i}^{(P)}}{C}\right)^{m}\left(\sigma_{0}+\beta E_{p} \varepsilon_{i}^{(P)}\right)
$$

where $\beta$ is the plastic strain hardening parameter, $\sigma_{0}$ is the initial, static yield point, $\dot{\varepsilon}_{i}^{(P)}$ is the plastic strain rate intensity, $C$ is the material parameter defining the effect of the plastic strain rate intensity, $m=1 / n$ is the material constant defining its sensitivity to the plastic strain rate, $\varepsilon_{i}^{(P)}$ is the plastic strain intensity and $E_{p}=E_{T} E /\left(E-E_{T}\right)$ is the material parameter depending on the modulus of plastic strain hardening $E_{T}=\partial \sigma_{Y} / \partial \varepsilon_{i}^{(P)}$ and Young's modulus $E$.

The Cowper-Symonds material deformation model is frequently used to perform simulations of dynamic processes using the FE method. Although the majority of material constants are determined using a classical tension test, problems are associated with the determination of the Cowper-Symonds constants $C$ and $n$. In a previous report (Bohdal and Kukielka, 2006), we established values of the Cowper-Symonds constants at different deformation rates. In this study, the values of the constants for aluminum alloy AA6111-T4 are taken as follows: $C=6500 \mathrm{~s}^{-1}$ and $n=4$, while $\rho=2720 \mathrm{~kg} / \mathrm{m}^{3}, \sigma_{0}=145 \mathrm{MPa}, E=76 \mathrm{GPa}, \nu=0.27, E_{T}=25 \mathrm{MPa}$, and $\beta=1$. 
To simulate the crack initiation and propagation in the material, we use a new constitutive model proposed by Xue and Wierzbicki (2013). The model covers the full range of plasticity until the onset of fracture. It is understood that the fracture initiation in uncracked solids is the ultimate result of a complex damage accumulation process induced by plastic deformations. Fracture can be predicted for complex loading paths that are not limited to a restricted loading in which the pressure is constant. The model also incorporates the coupling between the damage and the strain hardening function. We determined the model constants experimentally and presented them in (Bohdal and Kukielka, 2013).

\section{Results and discussion}

\subsection{Stress and strain analysis}

Figure 3 shows contours of the normal stresses $\sigma_{x x}, \sigma_{y y}$ and $\sigma_{z z}$. The stresses $\sigma_{y y}$ and $\sigma_{z z}$ show high compressive values in the region of the partially slit sheet, which is in contact with the knives. The compressive stresses occur because of pushing down of the knife surface on the sheet.
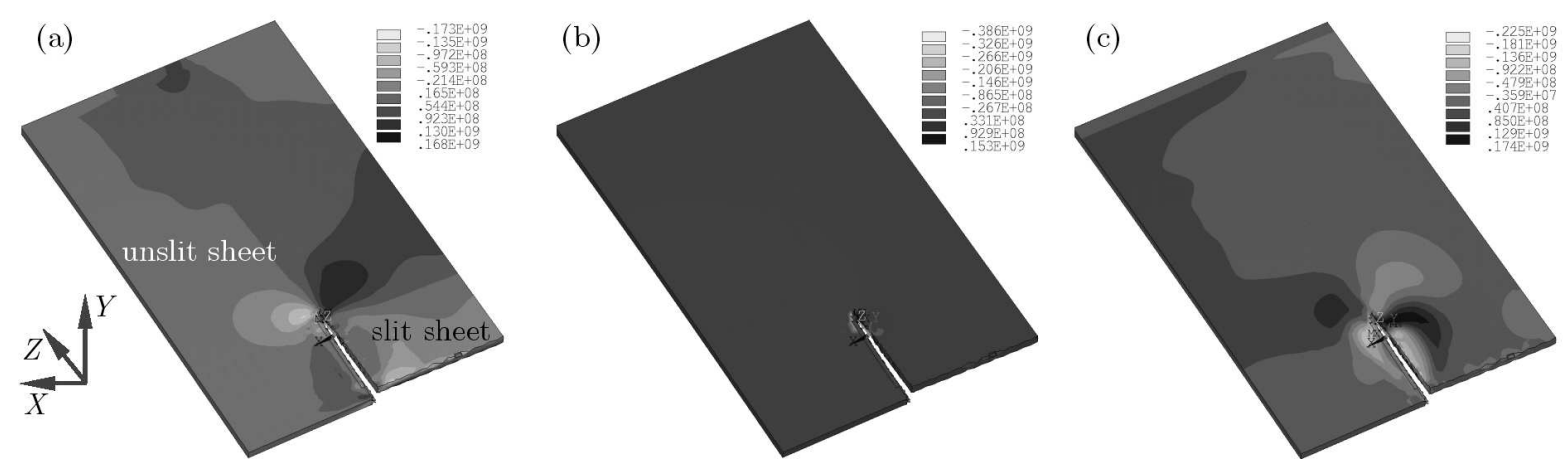

Fig. 3. Contours of the normal stresses; (a) $\sigma_{x x}$, (b) $\sigma_{y y}$ and (c) $\sigma_{z z}$ (25\% step time)

As the sheet slits, it moves tangentially to the blade. This causes the area of contact with the knife blade on the sheet to be inclined to the horizontal at an angle. The normal compressive stress is thus split into two components in the direction of the axes $Z$ and $Y$ contributing to the two normal stresses. In addition, it is observed that $\sigma_{x x}$ is also highly compressive in the same region of the partially slit sheet. The shear stress shows high values in the region where the sheet is expected to slit and the values drop down as the knives move away from the region (Fig. 4). The high shear stress and effective strain is caused by the shearing action of the two blades on either side of the sheet (Figs. 4 and 5). In the cutting area, material concentration takes place. After reaching the critical value of the effective strain, the process of integrity loss occurs (Fig. 5b). The results are close to those obtained by Gasiorek (2013a) during cutting of sheet metal bundles using a guillotine.

\subsection{Energy balance and slitting force analysis}

Figure 6 shows the energy curves obtained during the process. In shearing processes, the total increase of energy is nearly completely related to the increase of deformations in the integrity loss area (Gassiorek, 2013b; Bollen et al., 1989). The minimal increase of the kinetic energy results from the process of horizontal movement of a sheet during slitting (Fig. 6).

The evolution of the slitting force and its comparison to experimental measurement versus the upper knife penetration is shown in Fig. 7 with some deformed configurations of the sheet. 


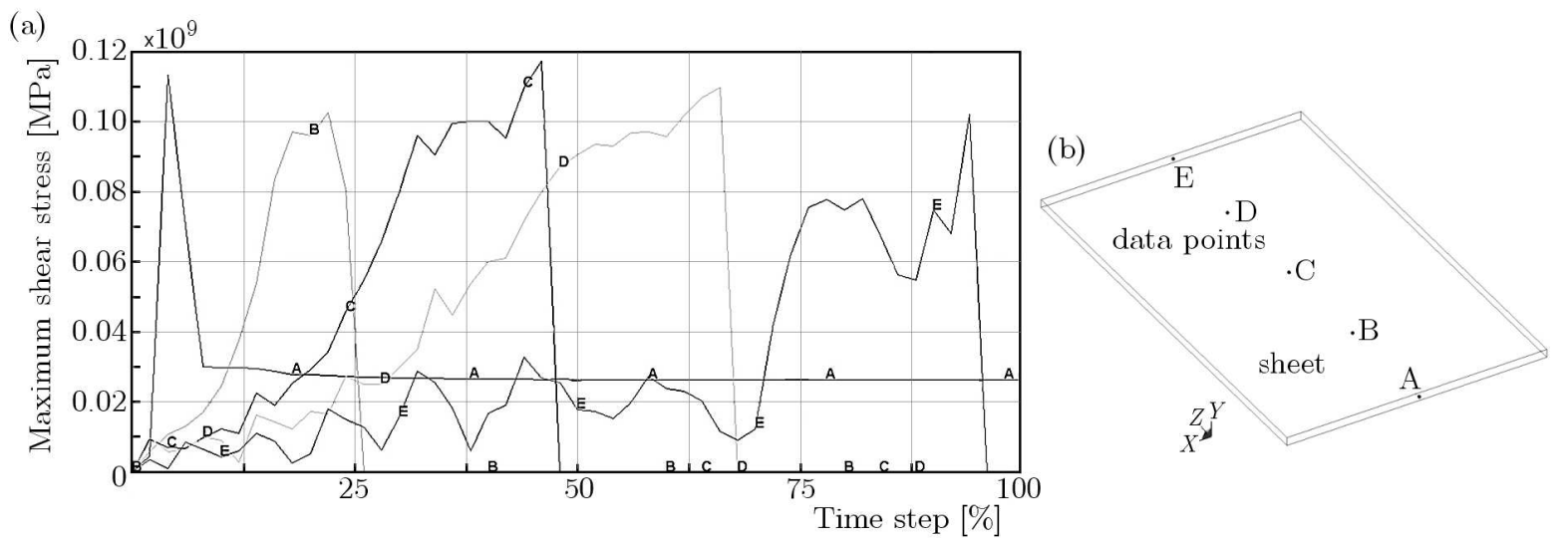

Fig. 4. Values of the maximum shear stresses measured in the shearing region during slitting
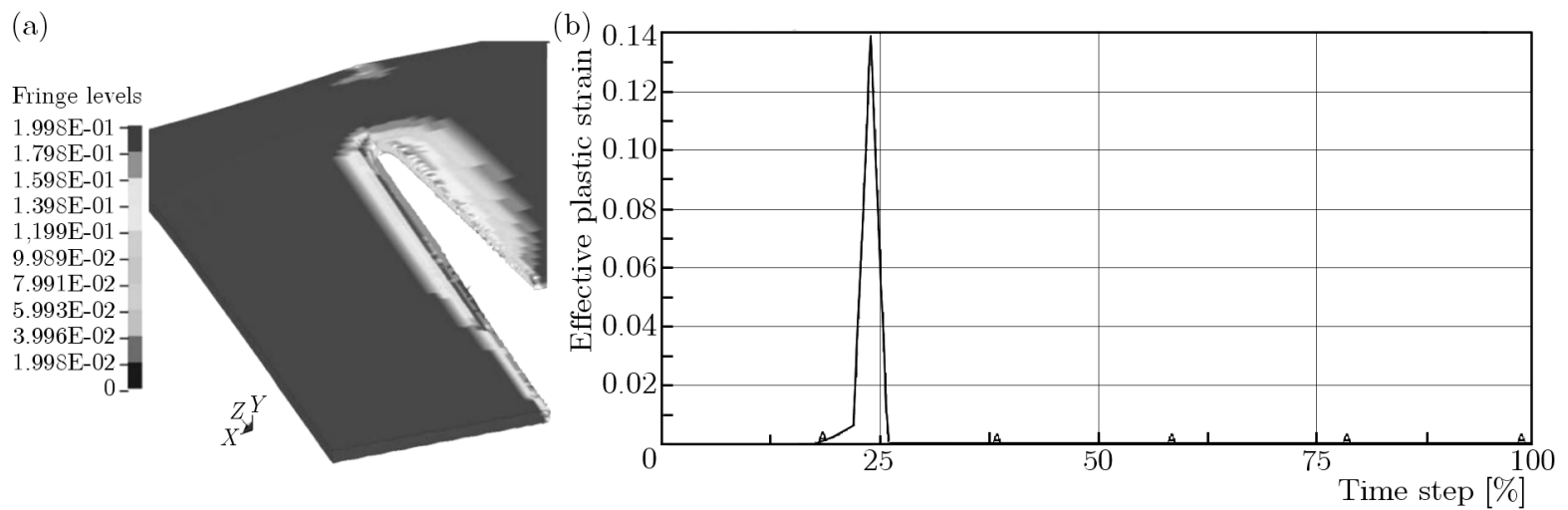

Fig. 5. (a) Contours of the effective plastic strain for $75 \%$ time step, (b) characteristics of the plasticization process for a selected mesh element describing the sheet in the cutting area up to the moment of decohesion

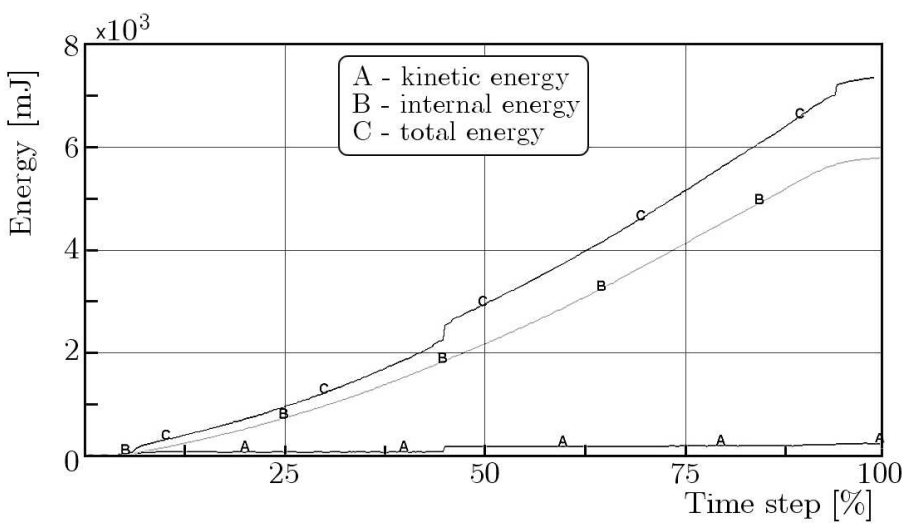

Fig. 6. Energy balance characteristics $\left[10^{-6} \mathrm{~kJ}\right]$

Three stages can be clearly observed. The first stage (AB) corresponds to a strong increase in the slitting force due to the increase of the bending forces in the sheet cross-section when the knives penetrate the sheet. The second stage extending from points $\mathrm{B}$ to $\mathrm{C}$ is characterized by an aquasi-constant slitting force $F_{\text {sta }} \approx 179 \mathrm{~N}$ which clearly indicates the steady state of this process stage. The last stage $(\mathrm{CD})$ of the slitting curve corresponds to the drastic decrease of the slitting force due to unstable propagation of the macroscopic crack along sheet thickness terminating the slitting operation. The experimental verification of the cutting force at steady state is shown in Fig. 7b. Values of forces obtained from numerical calculation show a little difference from the experimental results. This difference does not exceed $12 \%$. 

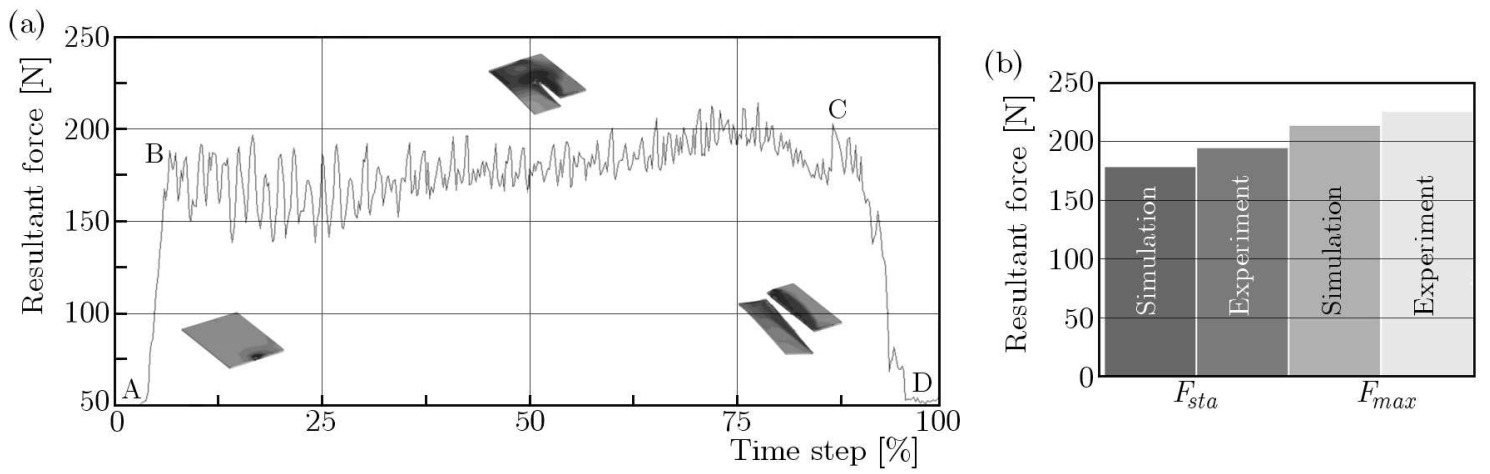

Fig. 7. A comparison of the resultant force from the experiment and the FEM simulation

\subsection{Sheared edge contours}

In this Section, we illustrate the capability of the present solution procedure to reproduce the effects of some process technological parameters such as slitting velocity and horizontal clearance on the quality of the sheared edge. In the industrial practice a high slitting sheared edge quality is required for all good products. In general, good sheared edge quality for cut sheets is indicated by smooth (burnished), clean, and straight edges with no edge wave (thin sheets), and most importantly, with no or minimal burr (Fig. 8). The area values (Fig. 8) are measured from simulations and experiments at different locations over the cut edge in the z-direction, averaged and presented in Figs. 9 and 10. The results are in good agreement with those obtained from the experiments, with an approximate error margin of $13 \%$. The experiments and simulations are executed for the slitting velocities $v=3,7,17,27,30$, and $32 \mathrm{~m} / \mathrm{min}$ and clearances $c=0.03$, $0.05,0.09,0.13$, and $0.15 \mathrm{~mm}$.
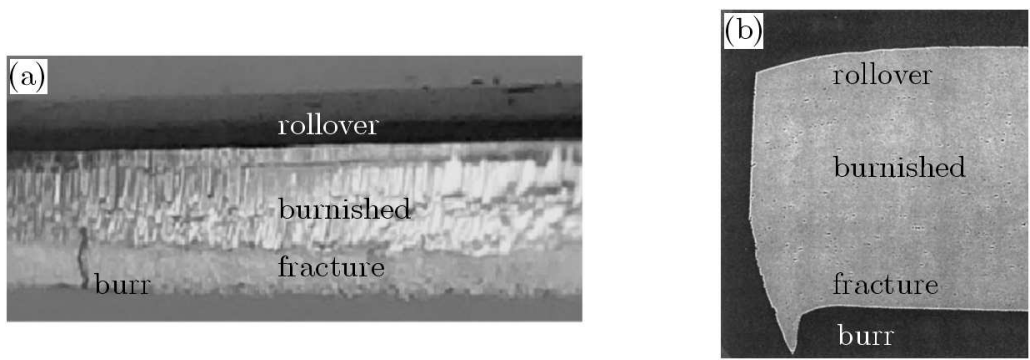

Fig. 8. Typical sheared edge profile with marked zones: (a) front view and (b) cross-sectional view
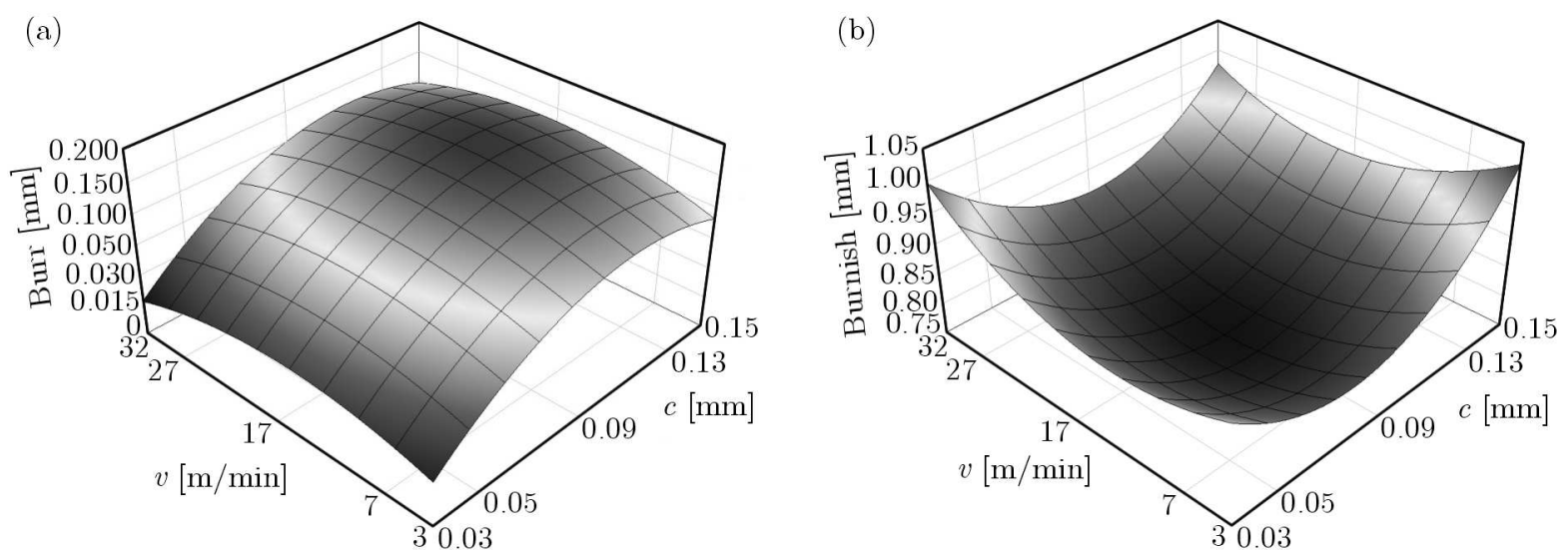

Fig. 9. Influence of the slitting velocity and clearance on the size of: (a) burr and (b) burnish zone 
(a)

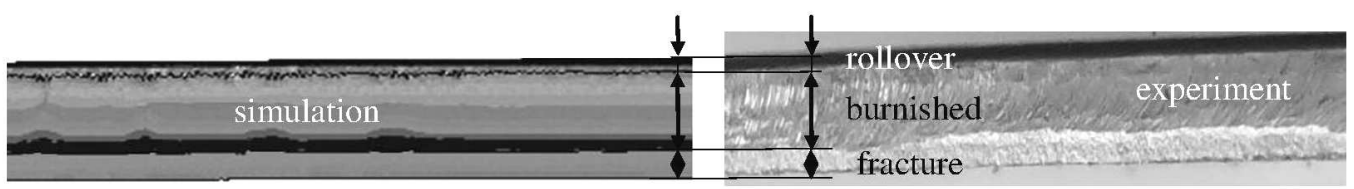

(b)

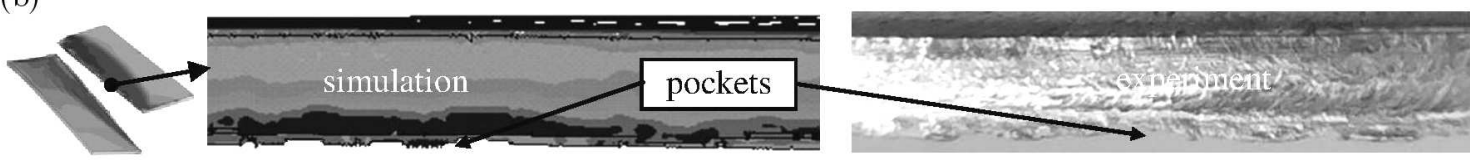

(c)
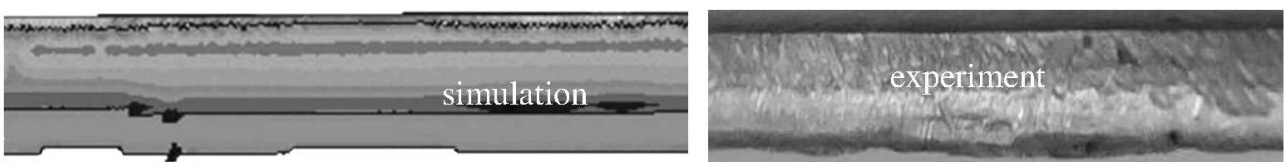

Fig. 10. Characteristic features of chosen sheared edges viewed by optical microscopy "Vision Engineering" and predicted by FE simulations at (a) $v=7 \mathrm{~m} / \mathrm{min}, c=0.05 \mathrm{~mm}$; (b) $v=17 \mathrm{~m} / \mathrm{min}$, $c=0.09 \mathrm{~mm}$, (c) $v=32 \mathrm{~m} / \mathrm{min}, c=0.09 \mathrm{~mm}$

Figure 9a shows experimental results of the effect of clearance and slitting velocity on burr height. The general trend seen in light alloys during another shearing process (blanking, guillotining) is for the burr height of the workpiece to increase with the increasing cutting clearance (Khadke et al., 2005). There are differences, though, in the relative rate of burr height increase with clearance. Increasing the clearance from $c=0.03 \mathrm{~mm}$ ( $2 \%$ of sheet thickness) to $c=0.09 \mathrm{~mm}$ $(6 \% t)$ increases the burr height for the analysed slitting velocities. When the clearance is set at $c>6 \% t$, the burr height decreases. The highest burrs are obtained when the middle range of velocities is used $(v=17 \mathrm{~m} / \mathrm{min})$ and when $c=6 \% t$. The height of the burrs in this case is non-uniform along the line of cutting, with some deep pockets that can serve as stress concentrators if stretching is applied parallel to this surface (Fig. 10b). Burrs can become separated from the cut part if there is a significant gradient of clearance along the shearing line. These local burrs are subjected to additional forces and torn off from the side of the sheared surface. When the clearance is set at $c=6 \% t$, the burnished zone is reduced (Fig. 9b) and the velocity effect is reinforced. A high zone value (approximately $66 \%$ of the sheet thickness) is obtained when clearances of $c=2 \% t$ and $c=10 \% t$ are used.

\section{Conclusions}

The objective of this paper is to present the modelling, analysis, and testing of a solution procedure for the analysis slitting process. Dynamic and damage effects, thermo-mechanical coupling and contact friction are taken into account. The procedure is implemented into a finite element code ANSYS LS-DYNA. The main conclusion is that most trends in the slitting process are qualitatively well described by the developed model. The model developed makes it possible to analyze the states of stresses and strains at any moment of the process and to analyse the causes of defects in the metal sheets after processing (deformation, edge wave, defects of the sheared edge, e.g., burrs). In the slitting, it is important to apply the required boundary conditions considering the dimensions of the sheet that is being cut. Special attention should be paid to the correct modeling of clamping of the sheet. This also means that 3D models cannot be simply replaced by $2 \mathrm{D}$ approximations.

The simulation results agree well with experiments, and the model can be used both to design the slitting process and as a support to the solution of practical problems. Actual investigations concern simulations of the slitting in which knives cannot be considered as rigid bodies. Further 
work is still to be done in order to introduce some other effects concerning the behavior of rolled metal sheets with damage induced anisotropy and spring-back effects.

\section{References}

1. Aggarwal S., Bhushan B., Katsube N., 2005, Three-dimensional finite element analysis of the magnetic tape slitting process, Journal of Materials Processing Technology, 170, 71-88

2. Bathe K.J., 1982, Finite Element Procedures In Engineering Analysis, Prentice-Hall, Englewood Cliffs, N.J.

3. Bohdal L., KukielKa L., 2006, The effect of selected material parameters on the stress and strain states in the process of cutting a sheet plate with circular cutters, Task Quarterley, 4, 391-400

4. Bohdal, L., Kukielka L., 2013, The modeling and numerical analysis of the shearing process with the regard of the geometrical and physical nonlinearity, Ecological Aspects of Applying New Technologies in Transport, 131-140

5. Bollen D., Deneir J., Aernoudt E., Muylle W., 1989, Shear cutting of PET film, Journal of Material Science, 24, 2957-2966

6. Gaef̨zia A., Gontarz S., Jasiński M., Mączak J., Radkowski S., Seńko J., 2012, Distributed system for monitoring of the large scale infrastructure structures based on analysis of changes of its static and dynamic properties, Key Engineering Materials, 518, 106-118

7. GąSIOREK D., 2013a, Modelling And Experimental Investigation Of Dynamic Processes Occurring During Cutting Lithograpic Plates Using Guillotines (in Polish), Wydawnictwo Politechniki Śląskiej, Gliwice

8. GasioneK D., 2013b, The application of the smoothed particle hydrodynamics (SPH) method and the experimental verification of cutting of sheet metal bundles using a guillotine, Journal of Theoretical and Applied Mechanics, 51, 4, 1053-1065

9. Gontarz S., RAdKowski S., 2012, Impact of various factors on relationships between stress and eigen magnetic field in a steel specimen, IEEE Transactions on Magnetics, 48, 3, 1143-1154

10. Khadke A., Ghosh S., Li M., 2005, Numerical simulations and design of shearing process for aluminum alloys, ASME Journal Manufacturing Science Engineering, 127, 3, 612-621

11. Kleiber M., 1985, Finite Element Method in Non-Linear Solid Mechanics, IPPT PAN, PWN, Warsaw-Poznań

12. Kukielka L., Geleta K., Kukielka K., 2012, Modelling of initial and boundary problems with geometrical and physical nonlinearity and its application in burnishing processes, Steel Research International, Special Edition, 14th International Conference on Metal Forming, 1375-1378

13. Kulakowska A., Kukielka L., 2008, Numerical analysis and experimental researches of burnishing rolling process with taking into account deviations in the surface asperities outline after previous treatment, Steel Research International, 2, 42-48

14. Liu C., Lu H., HuAng Y., 2005, Dynamic steady-state stress field in a web during slitting, ASME Journal Applied Mechanics, 72, 157-164

15. Lu H., Wang B., Iqbal J., 2001, Deformation in shear slitting of polymeric webs, Proceedings of the 6th International Conference on Web Handling, Stillwater, OK, June 10-13, 389-402

16. Ma J., Lu H., Li M., WANG B., 2006, Burr height in shear slitting of aluminum webs, ASME Journal Manufacturing Science Engineering, 128, 46-55

17. Meehan R. R., Burns S. J., 1998, Mechanics of slitting and cutting webs, Experimental Mechanics, 38, 2, 103-109

18. OrŁowska M., Czapp M., 2012, Numerical analysis of heat efficiency of the convective heat exchanger build with horizontal plates, Annual Set The Environment Protection, 14, 582-586 
19. Patyk R., Kukielka L., 2008, Optimization of geometrical parameters of regular triangular asperities of surface put to smooth burnishing, Steel Research International, 2, 642-647

20. SaAnouni K., 2006, Virtual metal forming including the ductile damage occurrence. Actual state of the art and main perspectives, Journal of Materials Processing Technology, 177, 19-25

21. Wisselink H.H., 2000, Analysis of guillotining and slitting: finite element simulations, Ph.D. Thesis, University of Twente, The Netherlands

22. Wisselink, H. H., Hú́tink J., 2004, 3D FEM simulation of stationary metal forming processes with applications to slitting and rolling, Journal of Materials Processing Technology, 148, 328-341

23. Xue L., Wierzbicki T., 2013, Verification of a new fracture criterion using LS-DYNA, 9th International LS-DYNA Users Conference, Simulation Technology, 3, 13-22

Manuscript received January 8, 2014; accepted for print December 30, 2014 